\title{
International recommendations for the assessment of autoantibodies to cellular antigens referred to as anti-nuclear antibodies
}

\author{
Nancy Agmon-Levin, ${ }^{1,2}$ Jan Damoiseaux, ${ }^{3}$ Cees Kallenberg, ${ }^{4}$ Ulrich Sack, ${ }^{5}$ \\ Torsten Witte, ${ }^{6}$ Manfred Herold, ${ }^{7,8}$ Xavier Bossuyt, ${ }^{9}$ Lucille Musset, ${ }^{10}$ \\ Ricard Cervera, ${ }^{11}$ Aresio Plaza-Lopez, ${ }^{12}$ Carlos Dias, $^{13}$ Maria José Sousa, ${ }^{14}$ \\ Antonella Radice, ${ }^{15}$ Catharina Eriksson, ${ }^{16}$ Olof Hultgren, ${ }^{17}$ Markku Viander, ${ }^{18}$ \\ Munther Khamashta, ${ }^{19}$ Stephan Regenass, ${ }^{20}$ Luis Eduardo Coelho Andrade, ${ }^{21}$ \\ Allan Wiik, ${ }^{22}$ Angela Tincani, ${ }^{23}$ Johan Rönnelid, ${ }^{24}$ Donald B Bloch, ${ }^{25}$ \\ Marvin J Fritzler, ${ }^{26}$ Edward K L Chan, ${ }^{27}$ | Garcia-De La Torre, ${ }^{28}$ \\ Konstantin N Konstantinov, ${ }^{29}$ Robert Lahita, ${ }^{30}$ Merlin Wilson, ${ }^{31}$ Olli Vainio, ${ }^{32}$ \\ Nicole Fabien, ${ }^{33}$ Renato Alberto Sinico, ${ }^{34}$ Pierluigi Meroni, ${ }^{35}$ Yehuda Shoenfeld ${ }^{1,2,36}$
}

\begin{abstract}
Handling editor Tore K Kvien
- Additional material is published online only. To view please visit the journal online (http://dx.doi.org/10.1136/ annrheumdis-2013-203863)

For numbered affiliations see end of article.
\end{abstract}

\section{Correspondence to} Professor Yehuda Shoenfeld, Center for Autoimmune Diseases, Sheba Medical Center, Tel Hashomer 52621, Israel; shoenfel@post.tau.ac.il

NA-L and JD contributed equally.

Received 30 April 2013 Revised 9 August 2013 Accepted 20 September 2013 Published Online First 14 October 2013
To cite: Agmon-Levin $\mathrm{N}$ Damoiseaux J, Kallenberg C, et al. Ann Rheum Dis 2014;73:17-23.

\section{ABSTRACT}

Anti-nuclear antibodies (ANA) are fundamental for the diagnosis of autoimmune diseases, and have been determined by indirect immunofluorescence assay (IIFA) for decades. As the demand for ANA testing increased, alternative techniques were developed challenging the classic IIFA. These alternative platforms differ in their antigen profiles, sensitivity and specificity, raising uncertainties regarding standardisation and interpretation of incongruent results. Therefore, an international group of experts has created recommendations for ANA testing by different methods.

Two groups of experts participated in this initiative. The European autoimmunity standardization initiative representing 15 European countries and the International Union of Immunologic Societies/World Health Organization/Arthritis Foundation/Centers for Disease Control and Prevention autoantibody standardising committee. A three-step process followed by a Delphi exercise with closed voting was applied.

Twenty-five recommendations for determining ANA (1-13), anti-double stranded DNA antibodies (14-18), specific antibodies (19-23) and validation of methods (24-25) were created. Significant differences between experts were observed regarding recommendations 24-25 ( $p<0.03)$. Here, we formulated recommendations for the assessment and interpretation of ANA and associated antibodies. Notably, the roles of IIFA as a reference method, and the importance of defining nuclear and cytoplasmic staining, were emphasised, while the need to incorporate alternative automated methods was acknowledged. Various approaches to overcome discrepancies between methods were suggested of which an improved bench-to-bedside communication is of the utmost importance. These recommendations are based on current knowledge and can enable harmonisation of local algorithms for testing and evaluation of ANA and related autoantibodies. Last but not least, new more appropriate terminologies have been suggested.

\section{INTRODUCTION}

Autoantibodies are a hallmark of autoimmunity, of which anti-nuclear antibodies (ANA) have taken the centre stage for the past 60 years. The term ANA is now outdated and even confusing as this historical label has come to encompass antibodies directed at various cellular compartments including nuclear constituents, components of the nuclear envelope, mitotic spindle apparatus, cytosol, cytoplasmic organelles and cell membranes. Detection of anti-cellular antibodies of the ANA family is pivotal to the diagnosis of many autoimmune diseases. ${ }^{12}$ Moreover, specific antibodies of the ANA family may present years before the appearance of overt disease, and for some conditions serological assays can provide useful information on the likelihood of clinical course or complications (eg, inflammatory myopathies, systemic lupus erythematosus (SLE)). Hence, the determination of ANA may enable the prediction, diagnosis and activity determination of certain autoimmune diseases. ${ }^{3} 4$

ANA detection by the indirect immunofluorescence assay (IIFA) was first described in 1950 by Coons and Kaplan. 5 This 'gold' standard technique is at present performed using HEp-2 cells or variants of this cell line (ie, HEp-2000). ${ }^{6}$ The IIFA requires a laborious process consisting of serial dilutions of positive sera, visual determination of the staining pattern, followed by a second test in which antigen specificity is determined. ${ }^{7}$ IIFA entails substantial technical expertise and while it is considered the 'gold' standard, it is only as good as the laboratory that performs this assay. Another limitation of IIFA is its lack of specificity. Indeed, depending on demographics, the population being studied, serum dilution, the cut-off used and other variables of this assay, up to $25 \%$ of sera from apparently healthy individuals can be ANA positive. ${ }^{9-14}$ Noteworthy, in the general population some individuals with a positive ANA test by IIFA do not have an autoimmune disease and are unlikely to develop one. 
In past decades, the demand for ANA testing has increased remarkably, raising the need for high throughput in service laboratories. These changes resulted in a technical evolution and the development of novel diagnostic platforms including the ELISA, addressable laser bead immunoassay, chemiluminescence immunoassay, fluorescent-enzyme immunoassay, line immunoassays $^{10}$ and others. ${ }^{8} 1516$ The latter are based on a limited number of purified and/or synthetic autoantigens. For details on the methodology see the supplementary material (available online only). The test characteristics of these novel assays are strongly distinct from the ANA-IIFA. Debates regarding ANA determination by different tools have recently resulted in a position statement of the American College of Rheumatology (ACR), claiming that IIFA is the 'gold' standard for ANA detection, and that alternative methods should demonstrate performance as good as IIFA (http://www.rheumatology.org/practice/ clinical/position/ana_position_stmt.pdf). This position statement is primarily based on the high sensitivity of IIFA for certain autoimmune diseases such as SLE and to a lesser extent systemic sclerosis (SSc). Moreover, it is strongly supported by the historical inclusion of the ANA, as performed by IIFA, as a criterion of certain diseases. Unfortunately, IIFA specificity and sensitivity is relatively low for other diseases (eg, inflammatory myopathies) and its technical limitations as detailed above are yet to be overcome.

Therefore, an international group of experts (laboratory specialists, scientists and clinicians) have developed a set of recommendations for the appropriate assessment and interpretation of anticellular antibodies of the ANA family determined by different methods.

\section{METHODS}

\section{Expert committees}

Two groups of experts participated in this initiative. The European autoimmunity standardization initiative (EASI) was formed a decade ago. This forum was assembled with the aims of improving the diagnosis of systemic autoimmune-rheumatic diseases (SARD), the rationale of autoantibody testing and to stimulate interactions between clinical and laboratory specialist while considering cost-effectiveness and novel techniques (http://www.easi-network.com/). In this study expert teams from 15 European countries, namely Italy, Germany, The Netherlands, France, UK, Sweden, Belgium, Finland, Norway, Switzerland, Austria, Spain, Ukraine, Portugal and Israel participated. Each national EASI team consists of clinicians, laboratory specialists and scientists, dedicated to the field of SARD. The final vote for each team was the average score of all participants from the same country.

The International Union of Immunologic Societies/World Health Organization/Arthritis Foundation/Centers for Disease Control and Prevention (IUIS/WHO/AF/CDC) committee for the standardisation of autoantibodies in rheumatic and related diseases (http://www.autoab.org) was established in the early 1980s, and is also affiliated with the ACR. The establishment of this group was primarily based on the recognised needs for reference human autoimmune sera that were critical for academic, clinical and industrial laboratories, as well as the need to communicate to the wider stakeholder community and to facilitate ongoing activities in continuing the mission in autoantibody standardisation. ${ }^{17}$

\section{Development of recommendations}

A three-step process was applied for creating these recommendations for the determination and interpretation of ANA and related antigen-specific antibody testing. First, 31 preliminary statements were generated by the EASI forum, based on previous algorithms from Italy, Germany, The Netherlands and the ACR. Following review of the literature, these recommendations were subjected to open comments by the EASI teams and grading by each EASI team on a scale of $1-5$ (ie, full agreement to no agreement, respectively). Thereafter, based on the results of the first stage, the EASI forum reformulated a new set of 25 recommendations. These were subjected to review and modifications by both the EASI teams and the IUIS/WHO/AF/CDC committee members.

In the third stage a Delphi exercise with closed voting followed. During this exercise the final 25 recommendations were separately voted on and given a score from 0 (absolutely no agreement with the recommendation) to 10 (maximal support for the recommendation). The means and SD scores of all participants were calculated to determine the level of agreement for each recommendation. Therefore, the highest agreement is defined by the highest mean score accompanied by the lowest SD. Separate scores for the voting by the EASI groups versus the IUIS members were assessed using the $t$ test $(p<0.05$ was considered significant).

\section{RESULTS}

Twenty-five recommendations were formulated in this study and divided into four subgroups. The first 13 recommendations were dedicated to the ANA test (1-13), five to the determination of anti-double stranded DNA antibodies (14-18) and five to the determination of specific antibodies including those directed to extractable nuclear antigens (ENA; 19-23). Recommendations 24-25 addressed the need for local validation of different methods. Each recommendation was graded by a Delphi voting score composed of voting by the EASI and the IUIS groups. Scores are presented as the mean \pm SD of the entire forum (table 1 ).

Significant statistical differences between the voting of the EASI teams and the IUIS members were observed only regarding recommendations 24 and 25 , which received the grades $8.3 \pm 3$ vs $10 \pm 0$ and $7.4 \pm 2.9$ vs $10 \pm 0$, respectively $(p<0.03)$.

The broad spectrum of nuclear and cytoplasmic staining patterns, in relation to antibody specificity and associated disease, is specified in table 2 .

\section{DISCUSSION}

In this study we formulated 25 recommendations intended to clarify and standardise clinical and technical aspects pertaining to the determination of ANA and related antigen-specific antibodies. In addition, key principles regarding terminology, discrepancies between methods and communication between laboratory and clinical specialists were addressed.

\section{Terminology}

The terms 'anti-nuclear antibodies' (ANA) and 'extractable nuclear antigens' (ENA) are no longer technically correct and do not cover the entire spectrum of relevant autoantibodies. 'ANA' may now detect antibodies directed against nuclear and non-nuclear elements (see recommendation 13), while 'ENA' may refer to antigens that are neither extractable nor nuclear. Therefore, one may suggest changing these outdated terms to appropriate ones, such as anticellular antibodies and specific antibodies, respectively. Such a change in nomenclature requires broad agreement within the medical community and an adjustment period, as most manuscripts and textbooks utilise these 'classic terms'. In the current manuscript we have used the 
Table 1 The recommendations for detection of autoantibodies to cellular components commonly referred to as ANA

Recommendation

Delphi score

$($ mean \pm SD)

1 The diagnosis of SARD requires a panel of specific laboratory tests (ie, ANA, anti-dsDNA and anti-ENA antibodies)

$9.6 \pm 0.9$

2 ANA, anti-dsDNA and anti-ENA testing should be included in the autoantibodies detection as part of the diagnostic work-up of SARD as well as some other autoimmune diseases (table 2)

3 The detection of ANA is the first level test for laboratory diagnosis of SARD

4 ANA testing is primarily intended for diagnostic purposes, and not for monitoring disease progression

5 The IIFA* is the reference method for ANA screening. Alternative assays can be used while keeping in mind that false negative and false positive ratio of these methods may be different. Thus, if the clinical suspicion is strong and the alternative method is negative, it is mandatory to perform IIFA

6 Diagnostic laboratories should specify the methods used for detecting ANA when reporting their results

7 Tests based on a (restricted) mixture of defined nuclear antigens should not be referred to as ANA test or ANA screen

$9.9 \pm 0.2$

$9.8 \pm 0.4$

$9.6 \pm 0.6$

$9.7 \pm 0.6$

$9.4 \pm 0.9$

$9.7 \pm 0.5$

8 Laboratories using in-house assays for detecting ANA, as well as anti-dsDNA and specific anti-ENA antibodies, should standardise each assay according to international standards (eg, WHO, CDC/IUIS)

9 For ANA screening by IIFA the conjugate should consist of fluorochrome-labelled anti-human IgG-specific secondary antibodies

$9.7 \pm 0.5$

A proper ANA-IIFA is dependent on reagents, equipment and other local factors, thus the screening dilution should be defined locally. An abnormal ANA should be the titre above the 95th percentile of a healthy control population. In general, a screening dilution of $1: 160$ on conventional HEp-2(000) substrates is often suitable for the detection of ANA in adult populations being evaluated for SARD

11 In the case of a positive ANA test, it is recommended that the pattern and the highest dilution to demonstrate reactivity be reported

12 ANA-IIFA patterns should be reported according to standardised terminology (table 2)

13 Besides nuclear patterns also cytoplasmic and mitotic apparatus patterns should be reported and specified when possible (table 2)

14 If ANA result is positive, testing for anti-dsDNA antibodies is advised when there is clinical suspicion of SLE

$9.7 \pm 0.4$

$9.5 \pm 1.0$

$9.7 \pm 0.4$

15 For anti-dsDNA antibody determination, the Farr assay and the CLIFT offer high clinical specificity. Alternative methods may yield lower specificity and, if so, it is recommended that positive results obtained by these methods be confirmed by CLIFT or Farr assay-and be reported separately

16 The method used for anti-dsDNA antibody detection should be included in the test result

17 Results of anti-dsDNA antibody detection should be reported quantitatively (or semiquantitatively for CLIFT)

18 For monitoring of SLE disease activity by quantitative determination of anti-dsDNA antibodies the same method should be used

$8.0 \pm 2.5$

19 In case of a positive ANA test during the diagnostic work-up (depending on pattern, titre and/or clinical setting), it is recommended to perform specific tests for anti-ENA antibodies (table 2)

20 For anti-ENA antibodies detection the method used should be reported. In the case of discrepancy with IIFA or with clinical suspicion, the use of an additional method should be considered

21 Results of assays for antibodies to specific ENA should be reported separately (including negative results); if the result of a screening assay is reported as negative, it is sufficient to communicate which ENA are present in that assay

22 Quantitative determination of positive anti-RNP antibodies is recommended in case of a clinical suspicion of mixed connective tissue disease

23 In case of high clinical suspicion the physician request for determination of antibodies to specific ENA should be granted, irrespective of the result of the ANA test. For instance, anti-Jo-1 antibodies for clinically suspected IM, anti-ribosomal P for SLE or anti-SS-A/Ro antibodies for congenital heart block/neonatal lupus/Sjögren's syndrome/subacute cutaneous lupus

24 Each laboratory should verify the recommended cut-off for kits used to determine ANA. It is recommended to use age and gender matched sera from healthy subjects from the general local population; cut-offs should be defined as the 95th percentile

25 Each laboratory should verify the recommended cut-off for kits used to determine anti-dsDNA and anti-ENA antibodies. It is recommended to use an adequate number of samples from patients with the appropriate autoimmune diseases, disease controls and healthy controls; cut-offs should be defined using ROC curve analysis

* See supplementary materials (available online only) for further information on the methodology of immunoassays.

ANA, anti-nuclear antibodies; CDC, Centers for Disease Control and Prevention; CLIFT, Crithidia luciliae immunofluorescence test; dsDNA, double-stranded DNA; ENA, extractable nuclear antigens; IIFA, indirect immunofluorescent assay; IM, inflammatory myopathies; IUIS, International Union of Immunologic Societies; RNP, ribonucleoproteins; ROC, receiver operating characteristic; SARD, systemic autoimmune rheumatic diseases; SLE, systemic lupus erythematosus; WHO, World Health Organization.

traditional term ANA and ENA, with the appreciation that they refer to a broader antigen specificity.

\section{Discrepancy between platforms}

The increasing demand for ANA tests led to the development of new automated methods for their detection, some of which probably represent the future of diagnostic serology. ${ }^{18}$ However, the inconsistencies between methods are currently a burden on those who perform and interpret these tests. For this reason, we have addressed the concepts of discordant results and communication between laboratory and clinical specialists. As a general rule, if discrepancy between methods is observed, especially in the setting of high clinical suspicion, another approved and validated diagnostic platform should be utilised. Notably, in certain cases, choosing another platform or deciding which method is preferred may require a collaborative assessment of clinicians and laboratory specialists. ${ }^{19}$ For example, the use of
anti-dsDNA antibody titration for monitoring SLE activity is better achieved by using a quantitative assay such as ELISA or the Farr assay, whereas at the time of the diagnosis, the immunofluorescence on Crithidia luciliae immunofluorescence test (CLIFT) or the Farr assays may offer the best specificity. ${ }^{20}$ Discrepancies between clinical assessment and laboratory results have also been reported. It is our recommendation that in cases of high clinical suspicion a physician request for the determination of specific antibodies should be granted, irrespective of the result of previous tests. For instance, specific antibodies directed against Jo-1, ribosomal P or SS-A/Ro may be detected in patients who are ANA negative by IIFA. In this regard, one may consider the possibility that for an individual patient serology may change over time. Another important issue at present concerns the use of ANA/anti-DNA testing as inclusion criteria for clinical trials that may require the use of several platforms balancing specificity versus sensitivity. 
Table 2 IIFA nuclear/cytoplasmic patterns detected on HEp-2 substrates and related antigens/diagnosis

\begin{tabular}{|c|c|c|}
\hline \multicolumn{3}{|l|}{ Most commonly recognised patterns } \\
\hline Nuclear patterns & Related antigens & Related diagnosis \\
\hline Homogeneous & dsDNA, histones, chromatin/nucleosomes, HMG & SLE, drug induced SLE/vasculitis, JIA \\
\hline Coarse speckled & U1-SnRNP, U2-6 snRNP (Sm), nuclear matrix & MCTD, SLE, Raynaud, SSc, SS, UCTD \\
\hline Fine speckled & SSA/Ro, SSB/La, Topo-1, common to many antigens & SLE, SS, SSC, IM, MCTD \\
\hline Centromere & Kinetochore: CENP-A, B, C, F & SSc (limited), Raynaud's \\
\hline Nucleolar & $\begin{array}{l}\text { PM/Scl, RNA-polymerase, URNP, U3-RNP, To/Th, B23 phosphoprotein/ } \\
\text { numatrin }\end{array}$ & SSc, Raynaud's, IM, overlap \\
\hline Cytoplasmic patterns & Related antigens & Related diagnosis \\
\hline Diffuse & RibP, Jo-1, other tRNA synthetases, SRP & SLE, IM \\
\hline Fine speckled & Jo-1, SRP, PDH (mitochondria) & IM, DM, PBC, interstitial lung disease \\
\hline \multicolumn{3}{|l|}{ Less commonly recognised patterns } \\
\hline Nuclear patterns & Related antigens & Related diagnosis \\
\hline Peripheral/rim or nuclear envelope & $\begin{array}{l}\text { Lamins, LAP1/2 gp210, nucleoporin p62; nuclear envelope and nuclear pore } \\
\text { complex antigens }\end{array}$ & SLE, RA, PBC, IM autoimmune liver diseases \\
\hline Dense fine speckled & DFS70/LEDGF-P75 & $\begin{array}{l}\text { Healthy subjects and other inflammatory } \\
\text { conditions }\end{array}$ \\
\hline Pleomorphic cell cycle speckled (PCNA) & $\begin{array}{l}\text { Auxiliary protein proliferating cell nuclear antigen: elongation factor of DNA } \\
\text { polymerase delta }\end{array}$ & SLE, lymphoproliferative diseases, SS \\
\hline Nucleolar (clumpy) & U3-SnRNP (fibrillarin) & SSC \\
\hline Multiple/few nuclear dots & Sp100, PML bodies, p80-coilin & PBC, CAH, SS \\
\hline $\begin{array}{l}\text { Centrosome/centriole (formerly:spindle } \\
\text { apparatus) }\end{array}$ & Enolase, ninein, pericentrin & SSc, Raynaud's, inflammatory disease \\
\hline MSA (mitotic spindle) & NuMA/centrophilin & $\begin{array}{l}\text { RA, inflammatory conditions; pneumonia } \\
\text { (mycoplasma) }\end{array}$ \\
\hline Cytoplasmic patterns & Related antigens & Related diagnosis \\
\hline Discrete speckled & $\begin{array}{l}\text { Endosome (early endosome antigen 1), GW/Processing bodies, multivesicular } \\
\text { bodies/lysosomes }\end{array}$ & $\begin{array}{l}\text { Neurological conditions, SS, SLE, RA, PBC, } \\
\text { UCTD }\end{array}$ \\
\hline Golgi complex & $\begin{array}{l}\text { Golgi proteins/golgins: giantin, golgin } 245 \text {, golgin } 110, \text { golgin } 97 \text {, golgin } 95 \text {, } \\
\text { others }\end{array}$ & $\begin{array}{l}\text { SLE, SS, RA, overlap syndromes, cerebellar } \\
\text { ataxia }\end{array}$ \\
\hline Cytoplasmic fibres & Actin, cytokeratin, tropomyosin, vimentin & $\begin{array}{l}\text { CAH, DM, infections and other inflammatory } \\
\text { diseases }\end{array}$ \\
\hline \multicolumn{3}{|c|}{$\begin{array}{l}\text { Within the many patterns that can be distinguished the ones specified in the upper part of the table are the most commonly recognised. The relationship between pattern and antigen } \\
\text { specificity may differ in certain conditions. Similarly, the specific antigens marked in bold are the most commonly detected by reflex testing, although other antigens may be of } \\
\text { importance in different clinical conditions. Less common pattern are specified in the lower part of this table. } \\
\text { CAH, chronic autoimmune hepatitis; CENP, centromere protein; DFS, dense fine speckled; DM, dermatomyositis; dsDNA, double-stranded DNA; GW, glycine-tryptophan; HMG, high mobility } \\
\text { group; IM, inflammatory myopathies; JIA, juvenile idiopathic arthritis; MCTD, mixed connective tissue disease; MSA, mitotic spindle; NuMA, nuclear mitotic apparatus; PBC, primary biliary } \\
\text { cirrhosis; PDH, pyruvate dehydrogenase; PM/Scl, polymyositis/scleroderma; RA, rheumatoid arthritis; RibP, ribosomal P protein; RNP, ribonucleoprotein; SLE, systemic lupus erythematosus; SRP, } \\
\text { signal recognition particle; SS, Sjögren's syndrome; SSc, systemic sclerosis; Topo-1, topo-isomerase 1; tRNA, transfer RNA; UCTD, undifferentiated connective tissue disease. }\end{array}$} \\
\hline
\end{tabular}

\section{Communication between the laboratory bench and the clinic}

This is an all-embracing issue detailed in many of our recommendations (ie, 7, 11, 12, 13, 14, 16, 19, 20 and 21). Notably, many clinicians are unaware of the specific method or the differences between methods used for ANA detection, and may erroneously apply the test characteristics of IIFA to other methods. Therefore, by and large the autoimmune laboratory should specify each method used. In addition, in the case of ANA detection by IIFA, titres and patterns obtained should be reported. If multiple methods are being used either for ANA, anti-dsDNA or other antigen-specific autoantibodies, the results obtained by each method should be reported separately. In addition, if further testing is advised (eg, the determination of anti-dsDNA in samples with high titre homogenous ANA by IIFA) a laboratory comment may be included as detailed below.

\section{The recommendations}

The first subgroup of recommendations (1-13) is dedicated to ANA screening at the first level of diagnosis. ${ }^{1}$ Most of our knowledge regarding ANA screening is based on data obtained by IIFA. As such, the prevalence of ANA-IIFA among SLE patients is expected to be above $95 \%$, while a lower prevalence may be detected using methods that rely on a limited number of antigens. ${ }^{78}$ Furthermore, ANA can be detected in patients with autoimmune, malignant or infectious diseases as well as in healthy subjects. Hence, the performance of any ANA assay is highly dependent on its pre-test probability, and interpretation of results should be governed by clinical circumstances. ${ }^{1}{ }^{18}$ In these recommendations, like others, we have recognised IIFA on HEp-2 (or HEp-2000) to be the reference method of choice for ANA screening. ${ }^{6}{ }^{21}$ However, we have also acknowledged the limitations of IIFA, including the time required for its performance and the necessity of highly qualified laboratory personnel. The latter raises the urgent need for training programmes on IIFA performing and autoimmune serology interpretation. ${ }^{22}$ In contrast, the alternative automated methods (see supplementary material, available online only) have come into common use, especially in high-throughput laboratories. These methods are 
simple to operate and may have the added value of better recognition of specific antibodies. Alas, they often lack sufficient sensitivity for certain diseases (ie, SLE and SSc) ${ }^{23-27}$ (recommendation 5). Considering the pro and cons, we have included the alternative assays in our recommendations as long as the platform being used is clearly specified (recommendation 6) and the result is not referred to as 'ANA test' or 'ANA screen', which are typically associated with ANA testing by IIFA (recommendation 7).

From the laboratory perspective, standardisation of IIFA is vital, and several variables of this method have been specified. The recommended substrate for ANA-IIFA derives from a human epithelial cell line (HEp-2 or HEp2000) and should contain a sufficient number of mitotic cells to enable adequate pattern recognition. Low positive control samples as well as an internal cut-off control, randomly distributed in distinct runs, may enable continuous training of technicians. The secondary detection antibody (conjugate) should consist of fluorochromelabelled anti-human IgG-specific antiserum (recommendation 9), either FITC or another validated new-generation fluorochrome. A special consideration, at a regular interval, to the fluorochrome potency and ultraviolet bulb strength was suggested. Another variable of ANA testing is the screening dilution of sera. ${ }^{6} 21$ Following review of the literature and many discussions among the committee experts, it was agreed that the optimal screening dilution must be defined locally, and the titre related to the 95th percentile of healthy controls considered the optimal screening dilution. In cases of uncertainty, while taking into consideration sensitivity, specificity and cost (ie, wells on slides, technician time, other reagents), we have pointed out that a screening dilution of $1: 160$ was often found to be the most suitable for the evaluation of adult patients. ${ }^{6}{ }^{21}$ In an international study, ANA was documented in $31.7 \%$ of healthy subjects at $1: 40$ serum dilution, $13.3 \%$ at $1: 80$ and $5.0 \%$ at $1: 160 .{ }^{21}$ Conversely, at $1: 160$ dilution the sensitivity for SARD is not perfect and a negative result does not exclude disease. Notably, no consensus could be achieved among experts regarding the screening dilution for children ( $<16$ years), for which some use a dilution of $1: 40$, or the need for further dilution once a positive result was obtained. Previously, it was generally accepted that individuals without autoimmune disease would present with lower serum levels while high-titre ANA are clinically more significant. ${ }^{12}$ However, there is ample evidence that low-titre ANA may be significant and that titres following the screening threshold of $1: 80$ or $1: 160$ have no bearing on diagnosis or disease activity. ${ }^{715}$

Patterns of ANA by IIFA reflect the topographic distribution of target autoantigens (recommendations 11,12$)^{28}$ and may convey significant information about antibody specificity (table 2). ${ }^{29}$ The role of cell cycle dynamics (ie, interphase, mitotic) in defining patterns is of great importance, especially for complex patterns related to autoantigens that exhibit a dynamic behaviour along the cell cycle, such as centromere protein (CENP)-F, NuMA-1 and topoisomerase- $1 .^{30-32}$ The recognition of both nuclear and cytoplasmic patterns enables 'reflex testing' (ie, testing for specific antibodies depending of the pattern) and improves the utility of the serological evaluation (recommendation 13). ${ }^{128}$

Antibodies directed against dsDNA are a key marker for diagnosis and follow-up of patients with SLE ${ }^{33} 34$ (recommendations 14-18). Theoretically, if ANA-IIFA is negative one should not proceed to defining anti-dsDNA antibodies, ${ }^{35} 36$ although if clinical suspicion of SLE is substantial anti-dsDNA antibodies assessment may be requested by the clinician. On the other hand, certain IIFA patterns (eg, homogenous) are suggestive of anti-dsDNA antibody (table 2). ${ }^{37}$ Regrettably, in most laboratories 'reflex testing' cannot be performed unless requested; thereby the addition of a note to the ANA-IIFA result should be considered (eg, 'This pattern may represent antibodies directed to DNA or chromatin components. Further tests are advised if there is a clinical suspicion of systemic lupus erythematosus'). The appropriate method of anti-dsDNA antibodies determination is still a matter of debate. The Farr assay and the CLIFT offer high clinical specificity (recommendation 15). ${ }^{35} 38$ In point of fact, the Farr assay is the one 'specific' for anti-dsDNA related to SLE diagnosis, owing to its ability to detect high avidity antibodies. However, both assays have substantial drawbacks. The Farr assay requires the use of radioisotopes, and may detect anti-dsDNA antibodies induced by drugs (ie, anti-tumour necrosis factors) ${ }^{39}$ while the CLIFT is not quantitative and is accompanied by relatively low sensitivity. Alternative methods for anti-dsDNA detection have recently been developed. Most of them provide high sensitivity but are hampered by lower specificity and lack of correlation with each other. ${ }^{38} 40$ Reflecting on these aspects, we have recommended that positive results obtained by these alternative methods be confirmed by CLIFT or Farr assay. Nonetheless, if discrepancy between methods is observed both results should be reported. In such cases a careful explanation should be provided by the laboratory and the final interpretation should take into consideration the clinical scenario and the ANA-IIFA pattern. Last but not least, for follow-up of anti-dsDNA titres a quantitative method (ie, not CLIFT) (recommendations 16, 17), preferably by the same method and performed by the same laboratory, is recommended (recommendation 18).

The identification of antibody specificity to nuclear and cytoplasmic antigens may further differentiate between diseases ${ }^{41-44}$ (table 2; recommendation 19). Although there is a clear correlation between IIFA patterns and antigen specificity (table 2), this association is not perfect and antigen specificity cannot be deduced from the IIFA pattern alone. Furthermore, disagreements between methods to detect specific antibodies and/or IIFA patterns were reported. ${ }^{4-46}$ In these cases an alternative method can be employed (recommendations 1, 20, 21). In addition, unlike anti-dsDNA, the coexistence of negative ANA by IIFA and seropositivity for specific antibodies by solid phase assay is not rare (ie, anti-SSA/Ro). ${ }^{47}$ Thus, a physician request to determine specific antibodies should be granted, irrespective of ANA-IIFA (recommendation 23). Notably, another aspect concerning anti-SSA/Ro antibody is the detection of its variants directed at Ro60 and Ro52/TRIM21, as some tests currently in use include only the Ro60 antigen. Detection of anti-SSA/Ro is recommended in different scenarios including counselling of patients with autoimmune disease who desire a pregnancy. The latter are mainly related to anti-Ro52/TRIM21 antibodies. Therefore, specifying the antigen used and evaluation of both anti-Ro52 and anti-Ro60 should be considered. The importance of specific antibody levels is still a matter of debate. In particular, the significance of anti- ribonucleoprotein levels (recommendation 22) data are lacking regarding levels determined by current methods in comparison to the historical haemaglutination test used for the mixed connective tissue disease criterion. ${ }^{48}$

The fourth subgroup of our recommendations addresses the issue of local validation of methods $(24,25)$. Both recommendations were fully accepted by the IUIS group and significantly less by the EASI teams. This divergence between experts may be explained by the different regulations applied in the different countries, namely in America and Canada local validation is frequently required by the authorities. Nevertheless, as the list of available methods is bound to increase, it is highly likely that in the years to come verification of assays will be an important feature of autoimmune laboratories worldwide. 


\section{CONCLUSIONS}

Defining anticellular antibodies is an essential component of the evaluation of patients with autoimmune diseases. The wide array of methods for their determination prompted questions and debate among laboratory personnel, clinicians, as well as co-authors of this manuscript. Therefore, in this study we have offered recommendations that are applicable and may facilitate the design of local algorithms for ANA detection. We have pointed out that the changes in the field of autoimmune diagnostics necessitate dedicated training of those who demand, perform and interpret the results. Therefore, in parallel to defining new autoantibodies and the development of new platforms an urgent need for training programmes for clinician, technicians and the industry has been recognised. Last but not least, further studies are required to address issues such as the local validation of platforms, significance of titres and patterns among different populations (ie, children), and different stages of diseases (ie, pre-clinical vs fully established; active vs quiescent) and the role of novel automated IIFA methods that may overcome many limitations of the manual form of this assay.

\section{Author affiliations}

${ }^{1}$ The Zabludowicz Center for Autoimmune Diseases, Sheba Medical Center, Tel Aviv, Israel

${ }^{2}$ Sackler Faculty of Medicine, Tel-Aviv University, Tel Aviv, Israel

${ }^{3}$ Central Diagnostic Laboratory, Maastricht University Medical Center, Maastricht, The Netherlands

${ }^{4}$ Department of Rheumatology and Clinical Immunology, University of Groningen, Groningen, The Netherlands

${ }^{5}$ University Leipzig, Institute for Clinical Immunology and Transfusion Medicine, Leipzig, Germany

${ }^{6}$ Department of Clinic for Immunology and Rheumatology, Medical School, Hanover, Germany

${ }^{7}$ Department of Internal Medicine 1, Rheumatology Unit, Innsbruck Medical University, Innsbruck, Austria

${ }^{8}$ Laboratory Medicine, University Hospitals Leuven, Leuven, Belgium

${ }^{9}$ Department of Microbiology and Immunology, KU Leuven, Leuven, Belgium

${ }^{10}$ Immunology Department, GH Pitie-Salpêtriere, Paris, France

${ }^{11}$ Department of Autoimmune Diseases, Hospital Clínic Barcelona, Catalonia, Spain

${ }^{12}$ Servicio de Inmunología, Hospital Universitario Pueta de Hierro. Majadahonda, Madrid, Spain

${ }^{13}$ Autoimmune Diseases Unit, Medicine Department, S. João Hospital, Porto, Portugal

${ }^{14}$ Autoimmunity and PreNatal Diagnostic Laboratory Unit, Fernando Fonseca Hospital, Porto, Portugal

${ }^{15}$ Institute of Microbiology, Azienda Ospedaliera Ospedale San carlo Borromeo, Milano, Italy

${ }^{16}$ Department of Clinical Microbiology, Clinical Immunology, Umeå University, Umeå, Sweden

${ }^{17}$ Department of Laboratory Medicine, Clinical Immunology, University Hospital of Orebro and Orebro University, Orebro, Sweden

${ }^{18}$ Department of Medical Microbiology and Immunology, University of Turku, Turku, Finland

${ }^{19}$ Lupus Research Laboratory Division of Women's Health, King's College London, London, UK

${ }^{20}$ Klinik für Immunologie, DIA UniversitätsSpital, Zürich, Switzerland

${ }^{21}$ Rheumatology Division, Universidade Federal de São Paulo', Sao Paulo, Brazil

${ }^{22}$ Department of Clinical Chemistry and Immunology, Statens Serum Institut, Copenhagen S, Denmark

${ }^{23}$ Rheumatology and Clinical Immunology Unit, Department of Clinical and Experimental Science, Spedali Civili and University of Brescia, Brescia, Italy ${ }^{24}$ Department of Immunology, Genetics and Pathology, Uppsala University, Uppsala, Sweden

${ }^{25}$ Division of Rheumatology, Allergy and Immunology, Massachusetts General Hospital and Harvard Medical School, Harvard, Massachusetts, USA

${ }^{26}$ Faculty of Medicine, University of Calgary, Calgary, Alberta, Canada

${ }^{27}$ Department of Oral Biology, University of Florida, Gainesville, Florida, USA

${ }^{28}$ Department Immunology and Rheumatology, Hospital General de Occidente and

University of Guadalajara, Guadalajara, Jal, Mexico

${ }^{29}$ Medicine/Rheumatology, UNM School of Medicine, New Mexico, USA

${ }^{30}$ Medicine and VP, Newark Beth Israel Medical Center UMDNJ, Newark, USA

${ }^{31}$ LSU School of Medicine, USA
${ }^{32}$ Department of Medical Microbiology and Immunology, Institute of Diagnostics, University of Oulu and NordLab Oulu, Oulu, Finland

${ }^{33}$ Department of Immunology, CH Lyon Sud, Pierre-Benite, France

${ }^{34}$ Clinical Immunology Unit and Renal Unit, Azienda Ospedaliera Ospedale San carlo Borromeo, Milano, Italy

${ }^{35}$ Division of Rheumatology, Ist G. Pini, Istituto Auxologico Italiano, University of Milan, Milan, Italy

${ }^{36}$ Sackler Faculty of Medicine, Incumbent of the Laura Schwarz-Kip Chair for Research of Autoimmune Diseases, Tel-Aviv University, Tel-Aviv, Israel

Correction notice This article has been corrected since it was published Online First. Additional collaborators have been included.

Collaborators JW Cohen Tervaert (Maastricht, The Netherlands); D Hamann (Amsterdam, The Netherlands); RHWM Derksen (Utrecht, The Netherlands); H Hooijkaas (Rotterdam, The Netherlands); I Klasen (Nijmegen, The Netherlands); P Limburg (Groningen, The Netherlands); R Smeenk (Amsterdam, The Netherlands); P van Daele (Rotterdam, The Netherlands); Kerstin Elvin (Stockholm, Sweden); Charlotte Dahle (Linköping, Sweden); Thomas Skogh (Linköping, Sweden); Jaakko Antonen (Tampere, Finland); Anna-Maija Haapala (Tampere, Finland); Marja Hietarinta (Turku, Finland); Teija Häkkinen (Helksinki, Finland); Arno Hänninen (Turku, Finland); Sakari Jokiranta (Helsinki, Finland); Anna Karjalainen (Oulu, Finland); Aino Laatikainen (Kuopio, Finland); Marjatta Leirisalo-Repo (Helsinki, Finland); Riitta Luosujärvi (Helsinki, Finland); Aaro Miettinen (Helsinki, Finland); Jari Siuro (Vantaa, Finland); Sune Sved (Vantaa, Finland); Tamara Tuuminen (Mikkeli, Finland); Timo Walle (Helsinki, Finland); Fátima Coelho (S. João Portugal); Maria José Santos (Barreiro Portugal); Mónica Bogas (Alto Minho Portugal); João P Ramos (Porto Portugal); João Faro-Viana (Lisboa Portugal); Nils Olivier Olsson (Dijon, France); Georges Chyderiotis (Lyon, France); Bach-Nga (Reims, France); Dr. Vincent Aubert (Lausanne, Switzerland); Clemens Dahinden (Bern, Switzerland); Eric Dayer (Sion, Switzerland); Françoise Mascart (Bruxelles, Belgium); Jean-Paul Tomasi (Louvain, Belgium); Martine Vercammen (Brussel, Belgium); Patrick Verschueren (Leuven, Belgium); Fréderic Houssiau (Louvain, Belgium); Bernard Lauwerys (Louvain, Belgium); Filip De Keyser (Gent, Belgium); Walter Fierz (Liechtenstein Switzerland); Ingmar Heijnen (Basel, Switzerland); Franco Keller (Bellinzona, Switzerland); Gerhard Müllner (Luzern, Switzerland); Stephan Regenass (Zürich, Switzerland); Pascale Roux-Lombard (Genève, Switzerland); Urs Wirthmüller (Bern, Switzerland); Boris Gilburde (Ramat-Gan, Israel); Rachel Levi (Beer Sheba, Israel); Daphna Lipinsky (Rehovot, Israel); Mira Barak (Haifa, Israel); Abu-Shakra Mahmud (Beer Sheva, Israel), Batia Donnenfeld (Tel Aviv, Israel); Ruth Fishel (Jerusalem, Israel), Bleiber Margalit (Tel Aviv, Israel); Mina Foguel (Rehovot, Israel); Kivity Shay (Ramat-Gan, Israel); Elena Csernok (Germany); Bernhard Schlüter (Germany); Bettina Becker (Germany); Rudolf Mierau (Germany); Torsten Witte (Hannover, Germany); Wolfram Seidel (Germany); Ingrid Frank (Germany); Arno Kromminga (Germany); Falk Hiepe (Berlin, Germany); Karsten Conrad (Dresden, Germany); Michael Sticherling (Germany); Guenter Steiner (Vienna, Austria), Chopyak Valentyna (Ukraine), Inmaculada Alcalá (Tenerife, Spain), Montserrat Alsina (Barcelona, Spain), María José Amengual (Barcelona, Spain), Ángela Carrasco (Madrid, Spain), Luis Fernández (Cáceres, Spain), María Ángeles Figueredo (Madrid, Spain), Carmen Gelpí (Barcelona, Spain), Juana Jiménez (Madrid, Spain), María Rosa Juliá (Palma de Mallorca, Spain), Marcos López-Hoyos (Santander, Spain), Lourdes Mozo (Oviedo, Spain), Cecilia Muñoz (Madrid, Spain), Francisco Javier Muñoz (Almería, Spain), Pilar Palomino (Madrid, Spain), Álvaro Prada (San Sebastián, Spain), Carmen Rodríguez Hernandez (Cádiz, Spain), Garbiñe Roy (Madrid, Spain), Estibaliz Ruiz (Barcelona, Spain), Luisa Vargas (Badajoz, Spain).

Contributors All authors have actively participated in this study as well as in writing the manuscript.

Competing interests None.

Provenance and peer review Not commissioned; externally peer reviewed.

\section{REFERENCES}

1 Solomon DH, Kavanaugh AJ, Schur PH. Evidence-based guidelines for the use of immunologic tests: antinuclear antibody testing. Arthritis Rheum 2002;47:434-44.

2 Agmon-Levin N, Shapira Y, Selmi C, et al. A comprehensive evaluation of serum autoantibodies in primary biliary cirrhosis. J Autoimmun 2010;34:55-8.

3 Arbuckle MR, McClain MT, Rubertone MV, et al. Development of autoantibodies before the clinical onset of systemic lupus erythematosus. N Engl J Med 2003:349:1526-33.

4 Eriksson $\mathrm{C}$, Kokkonen $\mathrm{H}$, Johansson $\mathrm{M}$, et al. Autoantibodies predate the onset of systemic lupus erythematosus in northern Sweden. Arthritis Res Ther 2011;13:R30.

5 Coons AH, Kaplan MH. Localization of antigen in tissue cells; improvements in a method for the detection of antigen by means of fluorescent antibody. J Exp Med 1950;91:1-13.

6 Meroni PL, Schur PH. ANA screening: an old test with new recommendations. Ann Rheum Dis 2010:69:1420-2. 
7 Kroshinsky D, Stone JH, Bloch DB, et al. Case records of the Massachusetts General Hospital. Case 5-2009. A 47-year-old woman with a rash and numbness and pain in the legs. N Engl J Med 2009;360:711-20.

8 Kivity S, Gilburd B, Agmon-Levin N, et al. A novel automated indirect immunofluorescence autoantibody evaluation. Clin Rheumatol 2012;31:503-9.

9 Fritzler MJ, Pauls JD, Kinsella TD, et al. Antinuclear, anticytoplasmic, and anti-Sjogren's syndrome antigen A (SS-A/Ro) antibodies in female blood donors. Clin Immunol Immunopathol 1985;36:120-8.

10 Li QZ, Karp DR, Quan J, et al. Risk factors for ANA positivity in healthy persons. Arthritis Res Ther 2011;13:R38.

11 Mahler M, Hanly JG, Fritzler MJ. Importance of the dense fine speckled pattern on HEp-2 cells and anti-DFS70 antibodies for the diagnosis of systemic autoimmune diseases. Autoimmun Rev 2012;11:642-5.

12 Mariz HA, Sato El, Barbosa SH, et al. Pattern on the antinuclear antibody-HEp-2 test is a critical parameter for discriminating antinuclear antibody-positive healthy individuals and patients with autoimmune rheumatic diseases. Arthritis Rheum 2011;63:191-200

13 Peene I, Meheus L, Veys EM, et al. Detection and identification of antinuclear antibodies (ANA) in a large and consecutive cohort of serum samples referred for ANA testing. Ann Rheum Dis 2001;60:1131-6.

14 Satoh M, Chan EK, Ho LA, et al. Prevalence and sociodemographic correlates of antinuclear antibodies in the United States. Arthritis Rheum 2012;64:2319-27.

15 Fritzler MJ. The antinuclear antibody test: last or lasting gasp? Arthritis Rheum 2011;63:19-22.

16 Fritzler MJ, Fritzler ML. The emergence of multiplexed technologies as diagnostic platforms in systemic autoimmune diseases. Curr Med Chem 2006;13:2503-12.

17 Chan EK, Fritzler MJ, Wiik A, et al. AutoAbSC.Org — Autoantibody Standardization Committee in 2006. Autoimmun Rev 2007;6:577-80.

18 Op De Beeck K, Vermeersch P, Verschueren $P$, et al. Detection of antinuclear antibodies by indirect immunofluorescence and by solid phase assay. Autoimmun Rev 2011;10:801-8.

19 Wiik AS, Gordon TP, Kavanaugh AF, et al. Cutting edge diagnostics in rheumatology: the role of patients, clinicians, and laboratory scientists in optimizing the use of autoimmune serology. Arthritis Rheum 2004;51:291-8.

20 Sherer Y, Gorstein A, Fritzler MJ, et al. Autoantibody explosion in systemic lupus erythematosus: more than 100 different antibodies found in SLE patients. Semin Arthritis Rheum 2004:34:501-37.

21 Tan EM, Feltkamp TE, Smolen JS, et al. Range of antinuclear antibodies in "healthy" individuals. Arthritis Rheum 1997;40:1601-11.

22 Melegari A, Bonaguri C, Russo A, et al. A comparative study on the reliability of an automated system for the evaluation of cell-based indirect immunofluorescence. Autoimmun Rev 2012;11:713-16.

23 Fritzler MJ, Wiik A, Tan EM, et al. A critical evaluation of enzyme immunoassay kits for detection of antinuclear autoantibodies of defined specificities. III. Comparative performance characteristics of academic and manufacturers' laboratories. J Rheumatol 2003:30:2374-81.

24 Molden DP, Nakamura RM, Tan EM. Standardization of the immunofluorescence test for autoantibody to nuclear antigens (ANA): use of reference sera of defined antibody specificity. Am I Clin Pathol 1984;82:57-66.

25 Russell AS, Johnston C. Relative value of commercial kits for ANA testing. Clin Exp Rheumatol 2003;21:477-80.

26 Tonutti E, Bassetti D, Piazza A, et al. Diagnostic accuracy of ELISA methods as an alternative screening test to indirect immunofluorescence for the detection of antinuclear antibodies. Evaluation of five commercial kits. Autoimmunity 2004;37:171-6.
27 Zandman-Goddard G, Gilburd B, Shovman 0, et al. The homogeneous multiplexed system - a new method for autoantibody profile in systemic lupus erythematosus. Clin Dev Immunol 2005:12:107-11.

28 Wiik AS, Hoier-Madsen M, Forslid J, et al. Antinuclear antibodies: a contemporary nomenclature using HEp-2 cells. J Autoimmun 2010;35:276-90.

29 Mahler M, Miyachi K, Peebles C, et al. The clinical significance of autoantibodies to the proliferating cell nuclear antigen (PCNA). Autoimmun Rev 2012;11:771-5.

30 Andrade LE, Chan EK, Peebles CL, et al. Two major autoantigen-antibody systems of the mitotic spindle apparatus. Arthritis Rheum 1996;39:1643-53.

31 Casiano CA, Humbel RL, Peebles C, et al. Autoimmunity to the cell cycle-dependent centromere protein $\mathrm{p} 330 \mathrm{~d} / \mathrm{CENP}-\mathrm{F}$ in disorders associated with cell proliferation. J Autoimmun 1995;8:575-86.

32 Tsoporis JN, Marks A, Kahn HJ, et al. S100beta inhibits alpha1-adrenergic induction of the hypertrophic phenotype in cardiac myocytes. J Biol Chem 1997;272:31915-21.

33 Hahn BH. Antibodies to DNA. N Engl J Med 1998;338:1359-68.

34 Ullal AJ, Reich CF 3rd, Clowse M, et al. Microparticles as antigenic targets of antibodies to DNA and nucleosomes in systemic lupus erythematosus. J Autoimmun 2011;36:173-80.

35 Kavanaugh AF, Solomon DH. Guidelines for immunologic laboratory testing in the rheumatic diseases: anti-DNA antibody tests. Arthritis Rheum 2002;47:546-55.

36 Kavanaugh A, Tomar R, Reveille J, et al. Guidelines for clinical use of the antinuclear antibody test and tests for specific autoantibodies to nuclear antigens. American College of Pathologists. Arch Pathol Lab Med 2000;124:71-81.

37 Servais G, Karmali R, Guillaume MP, et al. Anti DNA antibodies are not restricted to a specific pattern of fluorescence on HEp2 cells. Clin Chem Lab Med 2009;47:543-9.

38 Neogi T, Gladman DD, Ibanez D, et al. Anti-dsDNA antibody testing by Farr and ELISA techniques is not equivalent. I Rheumatol 2006;33:1785-8.

39 Atzeni $F$, Talotta R, Benucci $M$, et al. Immunogenicity and autoimmunity during anti-TNF therapy. Autoimmun Rev 2013;12:703-8.

40 Antico A, Platzgummer S, Bassetti D, et al. Diagnosing systemic lupus erythematosus: new-generation immunoassays for measurement of anti-dsDNA antibodies are an effective alternative to the Farr technique and the Crithidia luciliae immunofluorescence test. Lupus 2010;19:906-12.

41 Damoiseaux JG, Tervaert JW. From ANA to ENA: how to proceed? Autoimmun Rev 2006;5:10-17.

42 Benito-Garcia E, Schur PH, Lahita R. Guidelines for immunologic laboratory testing in the rheumatic diseases: anti-Sm and anti-RNP antibody tests. Arthritis Rheum 2004:51:1030-44.

43 Mehra S, Walker J, Patterson K, et al. Autoantibodies in systemic sclerosis. Autoimmun Rev 2013;12:340-54.

44 Villalta D, Imbastaro T, Di Giovanni S, et al. Diagnostic accuracy and predictive value of extended autoantibody profile in systemic sclerosis. Autoimmun Rev 2012;12:114-20.

45 Damoiseaux J, Boesten K, Giesen J, et al. Evaluation of a novel line-blot immunoassay for the detection of antibodies to extractable nuclear antigens. Ann NY Acad Sci 2005;1050:340-7.

46 Albon S, Bunn C, Swana G, et al. Performance of a multiplex assay compared to enzyme and precipitation methods for anti-ENA testing in systemic lupus and systemic sclerosis. J Immunol Methods 2011;365:126-31.

47 Bossuyt $X$, Luyckx A. Antibodies to extractable nuclear antigens in antinuclear antibody-negative samples. Clin Chem 2005;51:2426-7.

48 Shoenfeld Y, Cervera R, Gershwin ME. Diagnostic criteria in Autoimmune diseases. 1st edn. Humana Press, 2008. 


\section{International recommendations for the assessment of autoantibodies to cellular antigens referred to as anti-nuclear antibodies}

Nancy Agmon-Levin, Jan Damoiseaux, Cees Kallenberg, Ulrich Sack, Torsten Witte, Manfred Herold, Xavier Bossuyt, Lucille Musset, Ricard Cervera, Aresio Plaza-Lopez, Carlos Dias, Maria José Sousa, Antonella Radice, Catharina Eriksson, Olof Hultgren, Markku Viander, Munther Khamashta, Stephan Regenass, Luis Eduardo Coelho Andrade, Allan Wiik, Angela Tincani, Johan Rönnelid, Donald B Bloch, Marvin J Fritzler, Edward K L Chan, I Garcia-De La Torre, Konstantin N Konstantinov, Robert Lahita, Merlin Wilson, Olli Vainio, Nicole Fabien, Renato Alberto Sinico, Pierluigi Meroni and Yehuda Shoenfeld

Ann Rheum Dis2014 73: 17-23 originally published online October 14, 2013

doi: 10.1136/annrheumdis-2013-203863

Updated information and services can be found at:

http://ard.bmj.com/content/73/1/17

Supplementary Material

References

Email alerting service
These include:

Supplementary material can be found at:

http://ard.bmj.com/content/suppl/2013/10/14/annrheumdis-2013-2038 63.DC1

This article cites 47 articles, 7 of which you can access for free at: http://ard.bmj.com/content/73/1/17\#ref-list-1

Receive free email alerts when new articles cite this article. Sign up in the box at the top right corner of the online article.

\section{Notes}

To request permissions go to:

http://group.bmj.com/group/rights-licensing/permissions

To order reprints go to:

http://journals.bmj.com/cgi/reprintform

To subscribe to BMJ go to:

http://group.bmj.com/subscribe/ 